ESJ Social Sciences

\title{
Medición del Desempeño Organizacional en las Micro y Pequeñas Empresas del Sector Manufacturero en México
}

\author{
Dr. Ricardo Gallegos Murillo
}

Universidad Autónoma de Chihuahua, Mexico

Doi:10.19044/esj.2021.v17n27p58

Submitted: 20 March 2021

Accepted: 03 August 2021

Published: 31 August 2021
Copyright 2021 Author(s)

Under Creative Commons BY-NC-ND

4.0 OPEN ACCESS

Cite As:

Gallegos Murillo R. (2021). Medición del Desempeño Organizacional en las Micro y Pequeñas Empresas del Sector Manufacturero en México. European Scientific Journal, ESJ, 17 (27), 58. https://doi.org/10.19044/esj.2021.v17n27p58

\section{Resumen}

El desempeño organizacional facilita e impulsa el logro de los objetivos de la empresa a través de las personas. La tasa de mortalidad de las PYMES en México ha alcanzado el 4.5\%; una adecuada administración y gestión del desempeño pudiera contribuir a decrecer dicha tasa. El objetivo de esta investigación fue medir el desempeño organizacional en las micro y pequeñas empresas del sector manufacturero. El enfoque de la investigación fue de naturaleza cuantitativa, el diseño fue no experimental y transaccional, el muestreo fue no probabilístico. La población bajo estudio fueron las micro y pequeñas empresas del sector manufacturero (excluyendo el de alimentos) de la ciudad de Chihuahua, Chihuahua, México. En el período de tiempo comprendido entre los meses de noviembre del 2018 a mayo del 2019. Se estableció contacto con los grupos de interés y se procedió a recabar retroalimentación a través de llamadas telefónicas, correos electrónicos y visitas físicas a las empresas. Los resultados arrojaron que las micro y pequeñas empresas de este sector no cuentan con los conocimientos necesarios para implementar mediciones del desempeño. Los principales indicadores para medir el desempeño de las PYMES en la que coinciden la mayoría de los autores fueron: calidad, productividad, costos, participación del mercado, recursos humanos, capacidad instalada y satisfacción del cliente.

Palabras clave: Desempeño organizacional, indicadores de desempeño, Micro y pequeñas empresas 


\title{
Measurement of Organizational Performance in Micro and the Small Companies of the Manufacturing Sector in the City of Chihuahua, Mexico
}

\author{
Dr. Ricardo Gallegos Murillo \\ Universidad Autónoma de Chihuahua, Mexico
}

\begin{abstract}
Organizational performance facilitates and drives the achievement of company objectives through people. The mortality rate of SMEs in Mexico has reached $4.5 \%$; an adequate administration and performance management could contribute to decrease this rate. The objective of this research was to measure organizational performance in micro and small companies in the manufacturing sector. The research approach was quantitative in nature, the design was non-experimental and transactional, the sampling was nonprobabilistic. The population under study was the micro and small enterprises of the manufacturing sector (excluding the food sector) of the city of Chihuahua, Chihuahua, Mexico. In the period of time between the months of November 2018 to May 2019. Stakeholders were contacted and feedback was obtained through phone calls, emails and physical visits to the companies. The results showed that micro and small companies in this sector do not have the necessary knowledge to implement performance measurements. The main indicators to measure the performance of SMEs in which most of the authors agree were: quality, productivity, costs, market share, human resources, installed capacity and customer satisfaction.
\end{abstract}

Keywords: Organizational performance, performance indicators, micro and small size enterprises

\section{Introduction}

Las organizaciones se encuentran en un contexto particular el cual proporciona múltiples contextos que afectan a la organización y su desempeño, la evaluación del desempeño laboral es un proceso que se lleva a cabo de acuerdo con las particularidades de la organización y se utiliza como estrategia, tomando en cuenta la eficiencia, eficacia y la calidad de los objetivos de la organización en conjunto con la competitividad y las capacidades laborales, realiza una evaluación integral en base a los resultados de las aptitudes de los trabajadores y cómo afecta está en el cumplimiento de los objetivos y estrategias de la organización (Leyva, 2016). 
La evaluación del desempeño facilita información que sirve para decidir el ascenso de los colaboradores, remuneración o incentivos; para realizar esta evaluación se debe establecer una secuencia de pasos, iniciando por definir el puesto de trabajo, la evaluación del desempeño en función de del puesto y continuar con la respectiva retroalimentación (Alles, 2015).

Según Villar (2018) existen tres métodos técnicos significativos para evaluar el desempeño laboral, el primero es el de Key Performance Indicator (KPI), conocido como indicador clave, que mide las actividades y costos relacionados, efectuando comparaciones históricas, siendo un método sencillo y rápido para realizar un análisis de la fuerza laboral. Así mismo, Villar propone la técnica del análisis de costo beneficio y utilidad, la cual toma como fundamento la representación del personal de trabajo, los gastos y estrategias.

Matabanchoy, Álvarez, y Riobamba, (2018) señalan diversas técnicas de evaluación del desempeño laboral como la observación directa, que consiste en observar delicadamente a la persona a evaluar, registrando la información para el análisis; método de escala gráfica, el cual evalúa a los trabajadores por medio de parámetros que son definidos con antelación, precisando las aptitudes, actitudes, capacidades y cualidades dentro de la organización a través de tres tipos de escala, continua, semicontinua y discontinua.

En México la población de PYMES (Pequeñas y Medianas Empresas) en México era de 4.1 millones en 2010, lo que constituía el 52\% del PIB y $78.5 \%$ del empleo total. Es complicado concebir descripciones exactas de la distribución por tamaño de las empresas debido a las diferenciaciones entre las diferentes bases de datos. De acuerdo con el censo económico de 2008, el número total de unidades económicas en los tres principales sectores (comercio, servicios no financieros y manufactura) fue de 3,643,038 empresas de las cuales solo 5,944 eran grandes (es decir, 0.2\%). Por consiguiente, en $2008,3,638,982$ empresas en total se clasificaron como micro, pequeñas o medianas empresas (PYMES), lo que significa el $99.8 \%$ de todas las empresas. Las PYMES generan casi tres cuartas partes del empleo total en México.

Las micro, pequeñas y medianas empresas contribuyen en gran medida al producto interno bruto (PIB) de la mayoría de los países del mundo. Asimismo, se les atribuyen el desarrollo económico de las naciones, la creación constante de empleos y la incubación de innovaciones. La importancia ha sido reconocida a nivel internacional por académicos, emprendedores, políticos y otros grupos interesados en el desarrollo de los países. El mundo avanza velozmente hacia economías cuyo dinamismo está fuertemente vinculado a la existencia de una masa crítica de PYMES; cuanto mayor sea la proporción de PYMES en una economía, mayor será la flexibilidad de ésta, ya que dichas organizaciones se pueden adaptar rápidamente a los cambios en el entorno. Cada día se aumenta el interés por 
entender más y mejor la forma en la que se dinamizan las economías; en otras palabras, existe un interés creciente acerca de cómo emprender, dirigir y desarrollar (Filion, Cisneros, \& Mejía, 2011).

Las Pyme han enfrentado diversos problemas que han limitado su desarrollo. Por lo tanto, es esencial examinar la existencia de estas debilidades, de carácter estructural, y cómo influyen en su supervivencia y merman la competitividad. La globalización de los mercados y la aceleración del cambio tecnológico, unidas al pequeño hábito de gestión del conocimiento y del capital humano, así como el problema para la captación de capitales son, entre otros factores, algunos aspectos que obstaculizan y limitan el desarrollo de la microempresa (Martínez et al., 2008).

Los indicadores de demografía económica presentan diversos comportamientos por sector de actividad. Así, se señaló que en el sector manufacturero casi 7 de cada 10 negocios llegan con vida al cumplir el primer año. Para los sectores comercio y servicios privados no financieros este indicador se reduce a poco más de 6 de cada 10 que ingresan a la actividad económica (INEGI, 2015).

Sin embargo, el propiciar una evaluación demanda recursos, los beneficios de realizar la evaluación a veces elevan su costo, en la mayoría de los contextos (Guerra, 2007).

Por lo anteriormente expuesto y en base a la alta tasa de mortalidad de las PYMES, es preciso evitar que este tipo de organizaciones decaigan, lo cual puede ser mediante una apropiada administración y gestión del desempeño para que obtengan una operación eficiente. El objetivo de esta investigación fue medir el desempeño organizacional en las micro y pequeñas empresas del sector manufacturero de la ciudad de Chihuahua.

\section{Metodología}

La naturaleza de la investigación fue cuantitativa ya que las variables fue posible asignarles cantidades a través de valores numéricos. La investigación fue de tipo aplicada, debido a que abordó un problema ya detectado, en su etapa inicial, para posteriormente generar alternativas de solución.

El diseño de la investigación fue no experimental, ya que solo se observaron y midieron hechos que ya sucedieron o están sucediendo. No hubo manipulación por parte del investigador de variables. El diseño fue no experimental transeccional descriptiva ya que con la información obtenida se hicieron mediciones que indicaron la situación actual. La información se obtuvo en un periodo de tiempo específico.

La investigación fue de campo con apoyo bibliográfico. Fue transversal, la información fue recolectada en un solo momento basada en encuestas a gerentes y dueños de empresas. 
El tipo de investigación fue analítica se estudiaron las variables de manera general, no probabilística con una muestra de las micro y pequeñas empresas manufactureras de la ciudad de Chihuahua.

Se utilizó el muestreo por juicio, es una forma de muestreo por conveniencia, en el cual los elementos de la población se seleccionan con base en el juicio del investigador (Malhotra, 2008). Las variables evaluadas fue Desempeño Organizacional como dependiente y las variables independientes e indicadores fueron: 1) Rentabilidad (margen de utilidad operacional, margen de utilidad neta, rendimiento sobre los activos); 2) Calidad (sistema de calidad, mejora continua, calidad del producto, medición de la calidad); 3) Productividad (eficiencia, utilización); 4) Capacitación (horas de capacitación por empleado, capacitación técnica, capacitación interpersonal, capacitación para la resolución de problemas, correlación capacitación- competencias requeridas); 5) Tecnologías de información (TIC, gestión de la información); 6) Planeación estratégica (visión/misión, análisis FODA, planeación / objetivos estratégicos).

Se estructuró un cuestionario con 47 preguntas las cuales fueron cerradas para medir las variables en una escala del 0 al 4 donde: $0=$ No, nunca, $1=$ Muy poco, Raramente, 2= Poco, ocasionalmente, 3= Mucho, frecuentemente, $4=$ Bastante, muy frecuente y $5=\mathrm{Si}$, siempre.

La validación del instrumento se efectuó con una muestra piloto con 10 empresarios y paso seguido se siguieron las recomendaciones para mejorar el instrumento. El resultado del Alpha de Cronbach de este fue excelente según el criterio de George y Mallery pues el resultado fue de .984 de las 47 preguntas.

La población bajo estudio fueron las micro y pequeñas empresas del sector manufacturero (excluyendo el de alimentos) de la ciudad de Chihuahua, Chihuahua, México. En el período de tiempo comprendido entre los meses de noviembre del 2018 y mayo del 2019 se estableció contacto con los grupos de interés y se procedió a recabar retroalimentación a través de llamadas telefónicas, correos electrónicos y visitas físicas a las empresas.

Se utilizó la base de datos del SIEM (Sistema de Información Empresarial Mexicano) en las que existen 306 Micro y Pequeñas empresas que manufacturan bienes que no son comestibles. Después de esto se procedió a verificar por teléfono o visitas a ver cuáles empresas estaban todavía en operación y cuales eran manufactureras para depurar quedando la muestra de 32 empresas.

El tipo de muestreo fue no probabilístico y el método de muestreo por conveniencia en donde se contactaron alrededor de 135 empresas de las cuales accedieron a participar 32 empresas.

La selección de la muestra de las empresas dificultó la localización de varias de ellas ya que el sistema no estaba totalmente actualizado ya fuera por 
cambios de domicilio o por el cambio de la organización en enfocarse a servicios y dejar a un lado los procesos de manufactura.

Para facilitar el proceso se envió inicialmente el cuestionario vía electrónica al grupo de interés para ser contestado de una forma rápida. Debido a la casi nula respuesta a través de este medio, se optó por realizar las entrevistas de forma presencial. La poca disponibilidad de los administradores para poder atender las visitas solicitadas se debió a los múltiples compromisos laborales, lo que ocasionó constantes cancelaciones de las citas que dificultó la cantidad de empresas estudiadas. El poco tiempo que dedicaron al llenado del cuestionario derivó en la realización de una estrategia de aplicación del cuestionario de una forma más dinámica que facilito la conclusión del estudio.

\section{Resultados y discusión}

En los resultados se puede observar que las utilidades brutas y netas las monitorean de forma muy frecuente. Sin embargo, al cuestionarles sobre registros de los indicadores, solo un pequeño número contaba con ello. En el cuadro 1 se manifiesta un escaso grado de implantación de medibles de rendimientos sobre los activos en las empresas, ya que solo un $21.9 \%$ las medía frecuente o muy frecuentemente. Predomina con un 59.4\% los negocios que nunca o raramente monitorean los rendimientos sobre activos y con un $71.9 \%$ los que no cuentan o raramente lo hacen tener un objetivo de rendimiento sobre activos.

Solo el 53.2\% indicaron que consideran a los directivos como competentes en la compresión de la información financiera, el 56.3\% señaló llevar informes financieros que los soportan en la toma de decisiones. Así mismo el $59.4 \%$ de las empresas contestaron que toman acciones correctivas o preventivas para mejorar el desempeño financiero. 
Cuadro 1. Tabla distribución de frecuencias de rentabilidad

\begin{tabular}{|c|c|c|c|c|c|c|c|c|c|c|c|c|}
\hline \multirow[t]{2}{*}{ Rentabilidad } & \multicolumn{2}{|c|}{ Nunca } & \multicolumn{2}{|c|}{ Raramente } & \multicolumn{2}{|c|}{ Ocasionalmente } & \multicolumn{2}{|c|}{ Frecuentemente } & \multicolumn{2}{|c|}{$\begin{array}{c}\text { Muy } \\
\text { Frecuentemente }\end{array}$} & \multirow{2}{*}{$\begin{array}{c}\begin{array}{c}\text { Total } \\
\text { Frec } \\
\text { Acum }\end{array} \\
\text { Frec }\end{array}$} & \multirow{2}{*}{ 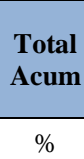 } \\
\hline & Frec & $\%$ & Frec & $\%$ & Frec & $\%$ & Frec & $\%$ & Frec & $\%$ & & \\
\hline $\begin{array}{l}\text { 1.1. Se monitorea } \\
\text { la utilidad bruta }\end{array}$ & 5 & 15.6 & 6 & 18.8 & 1 & 3.1 & 7 & 21.9 & 13 & 40.6 & 32 & 100 \\
\hline $\begin{array}{l}\text { 1.2. Se cuenta con } \\
\text { algún objetivo de } \\
\text { utilidad bruta }\end{array}$ & 11 & 34.4 & 5 & 15.6 & 0 & 0.0 & 7 & 21.9 & 9 & 28.1 & 32 & 100 \\
\hline $\begin{array}{l}\text { 1.3. Se monitorea } \\
\text { la utilidad neta }\end{array}$ & 3 & 9.4 & 7 & 21.9 & 2 & 6.3 & 8 & 25.0 & 12 & 37.5 & 32 & 100 \\
\hline $\begin{array}{l}\text { 1.4. Se cuenta con } \\
\text { algún objetivo de } \\
\text { utilidad neta }\end{array}$ & 7 & 21.9 & 6 & 18.8 & 1 & 3.1 & 7 & 21.9 & 11 & 34.4 & 32 & 100 \\
\hline $\begin{array}{l}\text { 1.5. Se monitorea } \\
\text { el rendimiento } \\
\text { sobre activos }\end{array}$ & 13 & 40.6 & 6 & 18.8 & 6 & 18.8 & 2 & 6.3 & 5 & 15.6 & 32 & 100 \\
\hline $\begin{array}{l}\text { 1.6. Se cuenta con } \\
\text { algún objetivo } \\
\text { sobre el } \\
\text { rendimiento sobre } \\
\text { los activos } \\
\end{array}$ & 19 & 59.4 & 4 & 12.5 & 0 & 0.0 & 5 & 15.6 & 4 & 12.5 & 32 & 100 \\
\hline $\begin{array}{l}\text { 1.7. Hay miembros } \\
\text { del personal y del } \\
\text { directorio que son } \\
\text { competentes y } \\
\text { comprenden la } \\
\text { función de los } \\
\text { procedimientos y } \\
\text { la información } \\
\text { financieros }\end{array}$ & 6 & 18.8 & 5 & 15.6 & 4 & 12.5 & 6 & 18.8 & 11 & 34.4 & 32 & 100 \\
\hline $\begin{array}{l}\text { 1.8. Hay informes } \\
\text { y estados } \\
\text { financieros para } \\
\text { apoyar una toma } \\
\text { de decisiones } \\
\text { efectiva y un buen } \\
\text { desempeño } \\
\text { 1.9. Se toman } \\
\text { acciones } \\
\text { correctivas o } \\
\text { preventivas para } \\
\text { mejorar la } \\
\text { situación } \\
\text { financiera de la } \\
\text { organización }\end{array}$ & 8 & 25.0 & 2 & 6.3 & 3 & 15.6 & 8 & 25.0 & 11 & 34.4 & 32 & 100 \\
\hline
\end{tabular}

Fuente: Elaboración propia (2018)

\section{Tecnologías de la Información y comunicación}

En el cuadro 2 se puede observar como el 50\% de las empresas encuestadas comentaron que las TIC unen frecuentemente a todos los miembros de la organización, el $59.40 \%$ comentó que el nivel actual de las tecnologías de la información es el adecuado para llevar a cabo las funciones. Solo el $25 \%$ de las empresas señaló que los empleados a menudo o muy 
frecuentemente laboran frente a computadoras o con dispositivos inalámbricos, también solo el $21.98 \%$ motiva el uso de los TIC. El $65.7 \%$ de las micro y pequeñas empresas no utiliza u ocasionalmente utiliza los sistemas de información para realizar los análisis de los ambientes externos e internos. El 62.5\% de empresas no llevan a cabo reuniones periódicas para revisar las últimas informaciones recopiladas. La información obtenida por las organizaciones en un $68.8 \%$ de los casos no la almacena en alguna base de datos y el $62.5 \%$ de ellas indicó no contar prácticamente con algún sistema de seguridad que impida que la información valiosa sea filtrada o mal empleada.

Cuadro 2. Tabla de frecuencias de Tecnologías de la información

\begin{tabular}{|c|c|c|c|c|c|c|c|c|c|c|c|c|}
\hline \multirow[t]{2}{*}{$\begin{array}{l}\text { Tecnologías de la } \\
\text { Información }\end{array}$} & \multicolumn{2}{|c|}{ Nunca } & \multicolumn{2}{|c|}{ Raramente } & \multicolumn{2}{|c|}{ Ocasionalmente } & \multicolumn{2}{|c|}{ Frecuentemente } & \multicolumn{2}{|c|}{$\begin{array}{c}\text { Muy } \\
\text { Frecuentemente }\end{array}$} & \multirow{2}{*}{$\begin{array}{c}\begin{array}{c}\text { Total } \\
\text { Frec } \\
\text { Acum }\end{array} \\
\text { Frec }\end{array}$} & \multirow{2}{*}{$\begin{array}{c}\begin{array}{c}\text { Total } \\
\text { Acum }\end{array} \\
\%\end{array}$} \\
\hline & Frec & $\%$ & Frec & $\%$ & Frec & $\%$ & Frec & $\%$ & Frec & $\%$ & & \\
\hline $\begin{array}{l}\text { 2.1. Dentro de su } \\
\text { organización, las TIC } \\
\text { unen a todos los } \\
\text { miembros de la } \\
\text { organización y a éstos } \\
\text { con los clientes, } \\
\text { proveedores, socios, } \\
\text { etc., en una forma } \\
\text { fácil e inmediata. }\end{array}$ & 5 & 15.6 & 7 & 21.9 & 4 & 12.5 & 5 & 15.6 & 11 & 34.4 & 32 & 100 \\
\hline $\begin{array}{l}\text { 2.2. En general, es } \\
\text { adecuado el nivel de } \\
\text { tecnologías de la } \\
\text { información para } \\
\text { llevar a cabo sus } \\
\text { funciones }\end{array}$ & 1 & 3.1 & 5 & 15.6 & 7 & 21.9 & 11 & 34.4 & 8 & 25.0 & 32 & 100 \\
\hline $\begin{array}{l}\text { 2.3. La mayor parte } \\
\text { de los empleados } \\
\text { trabajan normalmente } \\
\text { frente a una } \\
\text { computadora o con } \\
\text { dispositivos } \\
\text { inalámbricos }\end{array}$ & 8 & 25.0 & 11 & 34.4 & 5 & 15.6 & 5 & 15.6 & 3 & 9.4 & 32 & 100 \\
\hline $\begin{array}{l}\text { 2.4. La organización } \\
\text { motiva el uso } \\
\text { intensivo de las TIC } \\
\text { entre sus empleados } \\
\text { mediante la } \\
\text { capacitación, soporte, } \\
\text { disponibilidad de } \\
\text { equipos, etc. }\end{array}$ & 11 & 34.4 & 10 & 31.3 & 4 & 12.5 & 6 & 18.8 & 1 & 3.1 & 32 & 100 \\
\hline $\begin{array}{l}\text { 2.5. La organización } \\
\text { recaba de manera } \\
\text { sistemática } \\
\text { información sobre su } \\
\text { ambiente externo } \\
\text { (clientes, mercados, } \\
\text { productos sustitutos, } \\
\text { competidores, } \\
\text { proveedores, marcos } \\
\text { regulatorios, cambios } \\
\text { socioculturales, } \\
\text { económicos y } \\
\text { políticos, etc.) } \\
\end{array}$ & 8 & 25.0 & 5 & 15.6 & 8 & 25.0 & 4 & 12.5 & 7 & 21.9 & 32 & 100 \\
\hline
\end{tabular}




\begin{tabular}{|c|c|c|c|c|c|c|c|c|c|c|c|c|}
\hline $\begin{array}{l}\text { 2.6. La organización } \\
\text { recaba de manera } \\
\text { sistemática } \\
\text { información sobre su } \\
\text { ambiente interno } \\
\text { (producción, } \\
\text { finanzas, personal, } \\
\text { recursos físicos y } \\
\text { tecnológicos, ventas, } \\
\text { etc.). }\end{array}$ & 11 & 34.4 & 4 & 12.5 & 6 & 18.8 & 4 & 12.5 & 7 & 21.9 & 32 & 100 \\
\hline $\begin{array}{l}\text { 2.7. Se hacen } \\
\text { reuniones periódicas } \\
\text { para analizar las } \\
\text { últimas } \\
\text { informaciones } \\
\text { recopiladas y hacer } \\
\text { informes con } \\
\text { conclusiones y } \\
\text { recomendaciones } \\
\text { concretas. }\end{array}$ & 13 & 40.6 & 5 & 15.6 & 2 & 6.3 & 6 & 18.8 & 6 & 18.8 & 32 & 100 \\
\hline $\begin{array}{l}\text { 2.8. La información } \\
\text { obtenida por la } \\
\text { organización se } \\
\text { almacena en alguna } \\
\text { base de datos o } \\
\text { repositorio } \\
\text { compartido. }\end{array}$ & 15 & 46.9 & 4 & 12.5 & 3 & 9.4 & 5 & 15.6 & 5 & 15.6 & 32 & 100 \\
\hline $\begin{array}{c}\text { 2.9. La organización } \\
\text { cuenta con un sistema } \\
\text { de comunicación que } \\
\text { permite la difusión } \\
\text { selectiva de la } \\
\text { información } \\
\text { analizada, tal como } \\
\text { mensajería } \\
\text { electrónica, intranet, } \\
\text { etc. }\end{array}$ & 11 & 34.4 & 3 & 9.4 & 4 & 12.5 & 6 & 18.8 & 8 & 25.0 & 32 & 100 \\
\hline $\begin{array}{l}\text { 2.10. La organización } \\
\text { tiene sistemas de } \\
\text { seguridad que } \\
\text { impiden que la } \\
\text { información valiosa } \\
\text { se filtre o sea mal } \\
\text { empleada. }\end{array}$ & 13 & 40.6 & 5 & 15.6 & 2 & 6.3 & 5 & 15.6 & 7 & 21.9 & 32 & 100 \\
\hline
\end{tabular}

Fuente: Elaboración propia (2018)

En este apartado se puede observar que las preguntas con más resultados cargados de respuestas de nunca fueron la pregunta 2.8 donde no se almacenan los datos en algún medio electrónico, así como la pregunta 2.7 donde y 2.10 .

\section{Planeación estratégica}

En el cuadro 3 se evidencia que un $56.3 \%$ de las micro y pequeñas empresas cuentan con visión y misión y el mismo porcentaje mencionó que las mismas están alineadas con las metas de la organización, sin embargo, un $68.8 \%$ indicó que ocasional, raramente o nunca efectúan revisiones periódicas a la visión y misión. También el $68.7 \%$ comentó que no cuentan con procesos 
para explorar el ambiente externo. En lo concerniente al análisis interno para identificar las fortalezas y debilidades el $62.5 \%$ tampoco cuentan con algún proceso definido para llevarlo a cabo.

Solo el $37.5 \%$ de las organizaciones mencionó que cuentan con una estrategia formal, el $40.7 \%$ lleva sesiones de planeación estratégica y el 50\% tienen objetivos estratégicos definidos.

En cuanto al apoyo de los directivos para con la estrategia el $68.8 \%$ señaló que si lo tienen. En lo referente a los presupuestos adecuados para poder soportar los planes, el $62.5 \%$ de las empresas refirió no contar con ellos. El $71.9 \%$ de las empresas apuntó que nunca, rara u ocasionalmente cuentan con un proceso definido para monitorear los resultados de su planeación.

Cuadro 3. Frecuencias de Planeación Estratégica

\begin{tabular}{|c|c|c|c|c|c|c|c|c|c|c|c|c|}
\hline \multirow[t]{2}{*}{$\begin{array}{l}\text { Planeación } \\
\text { Estratégica }\end{array}$} & \multicolumn{2}{|c|}{ Nunca } & \multicolumn{2}{|c|}{ Raramente } & \multicolumn{2}{|c|}{ Ocasionalmente } & \multicolumn{2}{|c|}{ Frecuentemente } & \multicolumn{2}{|c|}{$\begin{array}{c}\text { Muy } \\
\text { Frecuentemente }\end{array}$} & \multirow{2}{*}{$\begin{array}{c}\begin{array}{c}\text { Total } \\
\text { Frec } \\
\text { Acum }\end{array} \\
\text { Frec }\end{array}$} & \multirow{2}{*}{ 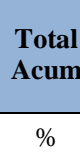 } \\
\hline & Frec & $\%$ & Frec & $\%$ & Frec & $\%$ & Frec & $\%$ & Frec & $\%$ & & \\
\hline $\begin{array}{c}\text { 3.1. Existen una } \\
\text { visión y misión } \\
\text { claras que } \\
\text { impulsan el } \\
\text { comportamiento } \\
\text { del personal } \\
\end{array}$ & 7 & 21.9 & 5 & 15.6 & 2 & 6.3 & 4 & 12.5 & 14 & 43.8 & 32 & 100 \\
\hline $\begin{array}{c}\text { 3.2. Esta la misión } \\
\text { alineada con las } \\
\text { metas y } \\
\text { direcciones de la } \\
\text { organización }\end{array}$ & 10 & 31.3 & 2 & 6.3 & 2 & 6.3 & 2 & 6.3 & 16 & 50.0 & 32 & 100 \\
\hline $\begin{array}{l}\text { 3.3. Se efectúan } \\
\text { revisiones } \\
\text { periódicas a la } \\
\text { visión y misión de } \\
\text { la organización }\end{array}$ & 14 & 43.8 & 5 & 15.6 & 3 & 9.4 & 4 & 12.5 & 6 & 18.8 & 32 & 100 \\
\hline $\begin{array}{l}\text { 3.4. Existe un } \\
\text { proceso } \\
\text { permanente para } \\
\text { explorar el entorno } \\
\text { a fin de considerar } \\
\text { amenazas y } \\
\text { oportunidades } \\
\text { potenciales }\end{array}$ & 12 & 37.5 & 5 & 15.6 & 5 & 15.6 & 4 & 12.5 & 6 & 18.8 & 32 & 100 \\
\hline $\begin{array}{l}\text { 3.5. Existe un } \\
\text { proceso definido } \\
\text { para evaluar las } \\
\text { fortalezas y } \\
\text { debilidades de la } \\
\text { organización } \\
\end{array}$ & 12 & 37.5 & 5 & 15.6 & 3 & 9.4 & 7 & 21.9 & 5 & 15.6 & 32 & 100 \\
\hline $\begin{array}{l}\text { 3.6. Cuenta la } \\
\text { organización con } \\
\text { una estrategia } \\
\text { formal }\end{array}$ & 9 & 28.1 & 8 & 25.0 & 3 & 9.4 & 8 & 25.0 & 4 & 12.5 & 32 & 100 \\
\hline $\begin{array}{l}\text { 3.7. Se llevan a } \\
\text { cabo sesiones de } \\
\text { planeación } \\
\text { estratégica }\end{array}$ & 13 & 40.6 & 4 & 12.5 & 2 & 6.3 & 7 & 21.9 & 6 & 18.8 & 32 & 100 \\
\hline
\end{tabular}




\begin{tabular}{|c|c|c|c|c|c|c|c|c|c|c|c|c|}
$\begin{array}{c}\text { 3.8. Se tienen } \\
\text { objetivos } \\
\text { estratégicos claros } \\
\text { y definidos }\end{array}$ & 9 & 28.1 & 2 & 6.3 & 5 & 15.6 & 5 & 15.6 & 11 & 34.4 & 32 & 100 \\
\hline $\begin{array}{c}\text { 3.9. Los directivos } \\
\text { apoyan la } \\
\text { estrategia de la } \\
\text { organización }\end{array}$ & 6 & 18.8 & 2 & 6.3 & 2 & 6.3 & 3 & 9.4 & 19 & 59.4 & 32 & 100 \\
\hline $\begin{array}{c}3.10 . \text { Se cuenta } \\
\text { con presupuestos } \\
\text { adecuados }\end{array}$ & 8 & 25.0 & 6 & 18.8 & 6 & 18.8 & 8 & 25.0 & 4 & 12.5 & 32 & 100 \\
\hline $\begin{array}{c}3.11 . \\
\text { Procedimientos } \\
\text { definidos para } \\
\text { monitorear los } \\
\text { resultados }\end{array}$ & 15 & 46.9 & 5 & 15.6 & 3 & 9.4 & 4 & 12.5 & 5 & 15.6 & 32 & 100 \\
\hline
\end{tabular}

Fuente: Elaboración propia (2018)

\section{Productividad}

En el cuadro 4, el 62.6\% de la empresas encuestadas manifestaron que nunca, raramente $\mathrm{u}$ ocasionalmente miden la productividad y al cuestionar sobre la medición de la eficiencia este porcentaje aumenta a un $65.7 \%$. El $56.3 \%$ indicó que utiliza en forma óptima las instalaciones.

En general la medición frecuente o muy frecuente de los índices de la relación de las unidades producidas respecto a los costos de producción fue de un $62.5 \%$ y la relación de los costos de la mano de obra respecto a la productividad resultó en un $53.1 \%$. El $50 \%$ de las micro y pequeñas citó que los recursos financieros son utilizados óptimamente. 
Cuadro 4. Frecuencias de productividad

\begin{tabular}{|c|c|c|c|c|c|c|c|c|c|c|c|c|}
\hline \multirow[t]{2}{*}{ Productividad } & \multicolumn{2}{|c|}{ Nunca } & \multicolumn{2}{|c|}{ Raramente } & \multicolumn{2}{|c|}{ Ocasionalmente } & \multicolumn{2}{|c|}{ Frecuentemente } & \multicolumn{2}{|c|}{$\begin{array}{c}\text { Muy } \\
\text { Frecuentemente }\end{array}$} & \multirow{2}{*}{$\begin{array}{c}\text { Total } \\
\text { Frec } \\
\text { Acum } \\
\text { Frec }\end{array}$} & \multirow{2}{*}{$\begin{array}{r}\begin{array}{r}\text { Total } \\
\text { Acum }\end{array} \\
\%\end{array}$} \\
\hline & Frec & $\%$ & Frec & $\%$ & Frec & $\%$ & Frec & $\%$ & Frec & $\%$ & & \\
\hline $\begin{array}{l}\text { 4.1. Se mide la } \\
\text { productividad de } \\
\text { la organización }\end{array}$ & 6 & 18.8 & 8 & 25.0 & 6 & 18.8 & 2 & 6.3 & 10 & 31.3 & 32 & 100 \\
\hline $\begin{array}{l}\text { 4.2. Se tienen } \\
\text { medibles de } \\
\text { eficiencia }\end{array}$ & 13 & 40.6 & 6 & 18.8 & 2 & 6.3 & 4 & 12.5 & 7 & 21.9 & 32 & 100 \\
\hline $\begin{array}{l}\text { 4.3. Se utilizan en } \\
\text { forma óptima las } \\
\text { instalaciones } \\
\text { físicas (edificios, } \\
\text { equipos, etc.) }\end{array}$ & 7 & 21.9 & 3 & 9.4 & 4 & 12.5 & 8 & 25.0 & 10 & 31.3 & 32 & 100 \\
\hline $\begin{array}{l}\text { 4.4 Cuáles son los } \\
\text { índices } \\
\text { comparativos de } \\
\text { costos y } \\
\text { resultados? }\end{array}$ & 5 & 15.6 & 7 & 21.9 & 5 & 15.6 & 6 & 18.8 & 9 & 28.1 & 32 & 100 \\
\hline $\begin{array}{l}4.5 \text { Cuál es la } \\
\text { relación entre la } \\
\text { unidad de } \\
\text { producto y el costo } \\
\text { de producir el } \\
\text { resultado? }\end{array}$ & 7 & 21.9 & 2 & 6.3 & 3 & 9.4 & 8 & 25.0 & 12 & 37.5 & 32 & 100 \\
\hline $\begin{array}{c}4.6 \text { Están } \\
\text { relacionados los } \\
\text { costos de los } \\
\text { miembros del } \\
\text { personal con su } \\
\text { productividad? }\end{array}$ & 7 & 21.9 & 2 & 6.3 & 6 & 18.8 & 5 & 15.6 & 12 & 37.5 & 32 & 100 \\
\hline $\begin{array}{l}4.7 \text { Se utilizan en } \\
\text { forma óptima los } \\
\text { recursos } \\
\text { financieros? }\end{array}$ & 6 & 18.8 & 3 & 9.4 & 7 & 21.9 & 4 & 12.5 & 12 & 37.5 & 32 & 100 \\
\hline
\end{tabular}

Fuente: Elaboración propia (2018)

\section{Calidad}

En el cuadro 5, se señala en lo referente a la calidad que la gran mayoría de las micro y pequeñas empresas del estudio apuntaron que en un $78.1 \%$ monitoreaban frecuente o muy frecuentemente la calidad de los productos o servicios. La proporción de empresas que cuentan con algún sistema de calidad fue de un $34.4 \%$. El $68.8 \%$ tampoco realiza encuestas o sondeos de los clientes para obtener las precepciones de la organización. El 62.6\% nunca, raramente u ocasionalmente tienen un proceso establecido para el análisis de soluciones de problemas. El porcentaje de organizaciones que no llevan a cabo algún monitoreo sobre la satisfacción y percepción de los clientes fue de $68.8 \%$ y el $59.4 \%$ nunca, rara u ocasionalmente cuentan con sistemas para la mejora continua. 
Cuadro 5. Frecuencias de calidad

\begin{tabular}{|c|c|c|c|c|c|c|c|c|c|c|c|c|}
\hline \multirow[t]{2}{*}{ Calidad } & \multicolumn{2}{|c|}{ Nunca } & \multicolumn{2}{|c|}{ Raramente } & \multicolumn{2}{|c|}{ Ocasionalmente } & \multicolumn{2}{|c|}{ Frecuentemente } & \multicolumn{2}{|c|}{$\begin{array}{c}\text { Muy } \\
\text { Frecuentemente }\end{array}$} & \multirow{2}{*}{$\begin{array}{r}\begin{array}{r}\text { Total } \\
\text { Frec } \\
\text { Acum }\end{array} \\
\text { Frec }\end{array}$} & \multirow{2}{*}{ 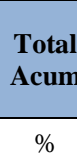 } \\
\hline & Frec & $\%$ & Frec & $\%$ & Frec & $\%$ & Frec & $\%$ & Frec & $\%$ & & \\
\hline $\begin{array}{l}\text { 5.1. Se monitorea } \\
\text { la calidad del } \\
\text { producto o } \\
\text { servicio }\end{array}$ & 1 & 3.1 & 4 & 12.5 & 2 & 6.3 & 9 & 28.1 & 16 & 50.0 & 32 & 100 \\
\hline $\begin{array}{c}\text { 5.2. La } \\
\text { organización } \\
\text { cuenta con algún } \\
\text { sistema de calidad }\end{array}$ & 13 & \begin{tabular}{|l|l} 
& 0.6
\end{tabular} & 4 & 12.5 & 4 & 12.5 & 5 & 15.6 & 6 & 18.8 & 32 & 100 \\
\hline $\begin{array}{c}\text { 5.3. Se realizan } \\
\text { encuestas o } \\
\text { sondeos adecuados } \\
\text { de los clientes para } \\
\text { obtener sus } \\
\text { percepciones de la } \\
\text { organización }\end{array}$ & 15 & \begin{tabular}{|l|l} 
\\
\end{tabular} & 6 & 18.8 & 1 & 3.1 & 4 & 12.5 & 6 & 18.8 & 32 & 100 \\
\hline $\begin{array}{l}5.4 \text { Se tiene algún } \\
\text { proceso } \\
\text { establecido para el } \\
\text { análisis de y } \\
\text { solución de } \\
\text { problemas }\end{array}$ & 16 & | 50.0 & 2 & 6.3 & 2 & 6.3 & 7 & 21.9 & 5 & 15.6 & 32 & 100 \\
\hline $\begin{array}{c}5.5 \text { Cuenta la } \\
\text { organización con } \\
\text { sistemas para el } \\
\text { mejoramiento } \\
\text { continuo }\end{array}$ & 13 & 40.6 & 3 & 9.4 & 3 & 9.4 & 9 & 28.1 & 4 & 12.5 & 32 & 100 \\
\hline
\end{tabular}

Fuente: Elaboración propia (2018)

\section{Capacitación}

En el cuadro 6, se puede apreciar las competencias para los puestos de trabajo en las micro y pequeñas empresas manufactureras de la ciudad de Chihuahua se tienen definidas frecuentemente en un 46.9\%. El 65.6\% de las empresas refirió que nunca, rara u ocasionalmente cuentan con algún plan de capacitación técnica para los empleados y dicho porcentaje aumenta a un 72\% respecto a planes de capacitación en aptitudes interpersonales para los trabajadores.

En el aspecto específico de capacitación a los empleados en el rubro de la resolución de problemas, el $78.2 \%$ de las empresas nunca, rara u ocasionalmente proveen este tipo de capacitación. Solo el $21.9 \%$ de las compañías señalaron haber proporcionado en el último año de manera formal capacitación a los empleados. 
Cuadro 6. Frecuencias de capacitación

\begin{tabular}{|c|c|c|c|c|c|c|c|c|c|c|c|c|}
\hline \multirow[t]{2}{*}{ Capacitación } & \multicolumn{2}{|c|}{ Nunca } & \multicolumn{2}{|c|}{ Raramente } & \multicolumn{2}{|c|}{ Ocasionalmente } & \multicolumn{2}{|c|}{ Frecuentemente } & \multicolumn{2}{|c|}{$\begin{array}{c}\text { Muy } \\
\text { Frecuentemente }\end{array}$} & \multirow{2}{*}{$\begin{array}{c}\text { Total } \\
\text { Frec } \\
\text { Acum } \\
\text { Frec }\end{array}$} & \multirow{2}{*}{$\begin{array}{r}\begin{array}{r}\text { Total } \\
\text { Acum }\end{array} \\
\%\end{array}$} \\
\hline & Frec & $\%$ & Frec & $\%$ & Frec & $\%$ & Frec & $\%$ & Frec & $\%$ & & \\
\hline $\begin{array}{c}\text { 6.1. Se tienen } \\
\text { definidas las } \\
\text { competencias para } \\
\text { cada puesto de } \\
\text { trabajo }\end{array}$ & 10 & 31.3 & 4 & 12.5 & 3 & 9.4 & 5 & 15.6 & 10 & 31.3 & 32 & 100 \\
\hline $\begin{array}{l}\text { 6.2. Cuenta la } \\
\text { organización con } \\
\text { algún plan de } \\
\text { capacitación } \\
\text { técnica para sus } \\
\text { empleados } \\
\end{array}$ & 12 & 37.5 & 5 & 15.6 & 4 & 12.5 & 5 & 15.6 & 6 & 18.8 & 32 & 100 \\
\hline $\begin{array}{l}\text { 6.3. Cuenta la } \\
\text { organización con } \\
\text { algún plan de } \\
\text { capacitación en } \\
\text { aptitudes } \\
\text { interpersonales } \\
\text { para sus } \\
\text { empleados }\end{array}$ & 15 & 46.9 & 6 & 18.8 & 2 & 6.3 & 6 & 18.8 & 3 & 9.4 & 32 & 100 \\
\hline $\begin{array}{l}\text { 6.4 Cuenta la } \\
\text { organización con } \\
\text { algún plan de } \\
\text { capacitación para } \\
\text { la resolución de } \\
\text { problemas }\end{array}$ & 19 & 59.4 & 6 & 18.8 & 0 & 0.0 & 4 & 12.5 & 3 & 9.4 & 32 & 100 \\
\hline $\begin{array}{l}\text { 6.5 Durante el } \\
\text { último año se le } \\
\text { dio capacitación a } \\
\text { los empleados en } \\
\text { donde queden } \\
\text { registros formales } \\
\text { de la misma }\end{array}$ & 20 & 62.5 & 4 & 12.5 & 1 & 3.1 & 2 & 6.3 & 5 & 15.6 & 32 & 100 \\
\hline
\end{tabular}

Fuente: Elaboración propia (2018)

\section{Discusión}

Los resultados de esta investigación exponen que las utilidades brutas y netas las monitorean de forma muy frecuente. Las TIC unen frecuentemente a todos los miembros de la organización, el 59.40\% comentó que el nivel actual de las tecnologías de la información es el adecuado para llevar a cabo las funciones. El 56.3\% de las micro y pequeñas empresas cuentan con visión y misión y el mismo porcentaje mencionó que las mismas están alineadas con las metas de la organización, sin embargo, un $68.8 \%$ indicó que ocasional, raramente o nunca efectúan revisiones periódicas a la visión y misión. En lo concerniente al análisis interno para identificar las fortalezas y debilidades el $62.5 \%$ tampoco cuentan con algún proceso definido para llevarlo a cabo.

El $62.6 \%$ comentó que nunca, rara u ocasionalmente miden la productividad y al cuestionar sobre la medición de la eficiencia este porcentaje aumenta a un $65.7 \%$. El $56.3 \%$ indicó que utiliza en forma óptima las 
instalaciones. En lo referente a la calidad la gran mayoría de las micro y pequeñas empresas del estudio apuntaron que en un $78.1 \%$ monitoreaban frecuente o muy frecuentemente la calidad de los productos o servicios. Gutiérrez (2013) en el estudio sobre el desempeño organizacional de microempresas en México menciona 4 indicadores para la medición del desempeño: tecnologías de la información y comunicación, calidad, capacitación y administración. El estudio se enfocó en el diagnóstico de 69 microempresas del sector turístico de la ciudad de Tapachula, Chiapas, México. Las dimensiones del estudio fueron las siguientes: 1) Tecnologías de Información y Comunicaciones (TIC); 2) Calidad; 3) Capacitación y 4) Administración Fiscal.

Los resultados de la investigación muestran que la gran mayoría, (90\%) opinó que las TIC ayudan en la empresa y reconocen que el uso de equipo de cómputo los ayuda a ser más eficientes. Sin embargo, 65\% considera que sí podría trabajar sin estas tecnologías, ya que anteriormente así lo hacían. Hay que reconocer que el uso y aplicación de las TIC en estos negocios es básico. En el aspecto de calidad, $57.5 \%$ señala contar con mecanismos que les permiten evaluar la calidad de su producto o servicio. Con relación a la capacitación, el $27.5 \%$ de las empresas no tuvieron curso alguno de capacitación, mientras que 50\% dijeron haber impartido entre ningún y un curso en el periodo de estudio. En lo referente al aspecto fiscal, el régimen tributario predominante es el de régimen general, que es utilizado por empresas con personalidad jurídica moral. Cabe mencionar que $57.5 \%$ de las empresas en la muestra se encontraban constituidas como sociedades. No hay, prácticamente, planeación fiscal alguna, simplemente se trabaja la parte obligatoria, que exigen las instancias gubernamentales.

La medición del desempeño frente a los estándares debería llevarse a cabo debidamente de forma anticipada para descubrir las desviaciones antes de que ocurran y evitarlas por medio de acciones adecuadas. El administrador que está alerta y tiene visión de futuro puede, en ocasiones, predecir posibles desviaciones de los estándares; sin embargo, en ausencia de tal capacidad éstas deben descubrirse tan pronto como sea posible" (Koontz, Weihrich, \& Cannice, 2012).

Gómez y López (2011) en el estudio rendimiento de las PYMES industriales de Puebla, utilizan el modelo de Quinn y Rohrbaugh para la medición del rendimiento de 33 PYMES.

Las variables de rendimiento utilizadas se basan en la clasificación propuesta por Quinn y Rohrbaugh (1983), que establecen un marco para el análisis organizacional y señalan que el criterio de eficiencia organizacional tiene tres ejes o dimensiones. La primera, relacionada con el enfoque de la organización, desde un punto de vista interno (basado en una visión micro sobre el buen entendimiento y el desarrollo del personal) a uno externo 
(énfasis a nivel macro del éxito de la empresa). La segunda, se centra en la estructura organizacional, haciendo hincapié desde la estabilidad hasta la flexibilidad. Y la tercera dimensión, se relaciona con los medios y fines organizacionales.

La función gerencial de control es la medición y corrección del desempeño para avalar que los objetivos de la empresa y los planes diseñados para alcanzarlos se alcancen (Koontz, Weihrich, \& Cannice, 2012).

El Instituto para el Fomento a la Calidad Total, A.C., en el artículo Modelo Nacional para la Competitividad Micro y Pequeñas Empresas (2013) menciona que el Modelo Nacional para la Competitividad Micro y Pequeñas Empresas es una herramienta directiva que tiene por objeto provocar una reflexión sobre el Modelo de Negocio que incurra en el desarrollo de capacidades de la organización y con éstas en la generación de ventajas competitivas en las micro y pequeñas empresas que lo aplican.

El modelo responde a la necesidad de fortalecer la capacidad de administración de las micro y pequeñas empresas: para que ésta sirva como una plataforma que soporte la profesionalización, crecimiento y sustentabilidad.

Los resultados responden a la necesidad de mantener una visión integral de los indicadores clave del negocio para asegurar la ejecución de la estrategia y de la propuesta de valor, evaluar el desempeño y capitalizar el aprendizaje que esta evaluación genera para tomar decisiones que soporten la eficiencia y crecimiento del negocio.

Molina, López (2011) señala que la actual propuesta de diagnóstico para Pymes en México tiene precedentes en los trabajos de St-Pierre y Delisle (2006) de cómo el benchmarking ayuda a las Pymes a mejorar las operaciones y desempeño financiero, con la implementación de un sistema de diagnóstico experto para valuar el desempeño de cientos de Pymes en las diferentes regiones de la provincia de Quebec, Canadá.

\section{Conclusiones y recomendaciones}

Los indicadores clave que resultaron mas significativos en las PyME del sector servicios de la ciudad de Chihuahua fueron la rentabilidad, las Tecnologías de Información y Comunicaciones, Planeación estratégica, rentabilidad, productividad, capacitación y calidad, jugando un factor clave las TIC seguido de la calidad y capacitación.

La problemática del sector PyME se puede visualizar desde dos puntos de vista, uno los factores externos donde los empresarios no tienen intervención como las políticas públicas y segundo los factores internos que se relacionan directamente con su propia gestión donde su participación es necesaria. Los inconvenientes internos del sector PyME generalmente son derivados de una administración deficiente y la falta de habilidades de gestión. 
Para la permanencia de las empresas del sector PyME se deben desarrollar frecuentemente diferentes estrategias administrativas y una de gran significancia es la medición del desempeño, en donde se pueden estableces procesos a través de diferentes metodologías que promuevan nuevos conocimientos para apoyar la mejora continua de la organización.

La estrategia que utilizan las empresas para posicionarse en el mercado es un factor que juega un papel significativo en su rendimiento. Por este motivo el establecimiento de indicadores clave de medición del desempeño en la organización cobran una mayor relevancia en el caso de las PYME, ya que por medio de estos de puede lograr ser mas eficientes y competitivas a nivel nacional e internacional.

En el análisis bibliográfico efectuado en este estudio se encontró que los principales indicadores para medir el desempeño de las PYMES en la que coinciden la mayoría de los autores son: calidad, productividad, costos, participación del mercado, recursos humanos, capacidad instalada y satisfacción del cliente.

Para que las PYMES sean exitosas en un ambiente competitivo es recomendable que apliquen algún modelo de medición del desempeño que los guie hacia una mejor toma de decisiones.

\section{References:}

1. Cossman, E. J. (2010) http:/allinonestep.com. Soluciones web para MiPymes http://allinonestepicom/definicion-y-características-de-mipyme. (15 marzo 2014)

2. Gómez, A. y López, M. (2011) Importancia de los factores competitivos en el rendimiento de la Pyme industrial de Puebla: Una aproximación empírica.

http://congreso.investiga.fca.unam.mx/docs/anteriores/xvi/docs/1N.p df (25-02-2014)

3. Gutiérrez, M. (2013) Desempeño Organizacional de Microempresas en México

http://url?sa=t\&rct=j\&q=\&esrc=s\&frm $=1 \&$ source $=$ web $\&$ cd $=6 \& v e d=$ OCEAQFjAFahUKEwihrrGkmpzHAhXOE5IKHfBxBbY\&url=http\% 3A\%2F\%2Feujournal.org\%2Findex.php\%2Fesj\%2Farticle\%2Fdown load\%2F1886\%2F1828\&ei=4WLHVeG5Gs6nyATw45WwCw\&usg $=$ AFQjCNFYWeIWBh6vayJyuJP3NdlvxINULA

4. Filion, L., Cisneros, L., \& Mejía, J. (2011). Adminsitracion de PyMES: Emprender, dirigir, desarrollar empresas. Mexico: Prentice Hall.

5. Heredia L., García D. y Bastida F. (2008). Los sistemas de control de gestión y la innovación: su efecto sobre el rendimiento de las pymes (Yucatán. 
http://www.saber.ula.ve/bitstream/123456789/26629/1/articulo11.pdf (25-02-2014)

6. Hitt, Black y Porter (2006) . Administracion. Pearson, Prentice Hall.

7. INEGI. (2015). Instituto Nacional de Estadistica y Geografia. Obtenido de http://www.inegi.org.mx/

8. Instituto para el fomento a la calidad total, A.C., (2013). Modelo nacional para la competitividad micro y pequeñas empresas. htpp://competitividad.org.mx/images/stories/modelo-pnc-myp- (2502-2014)

9. Koontz, H., Weihrich, H., \& Cannice, M. (2012). Administración una perspectiva global y empresarial. México: Mc Graw Hill.

10. Leyva, C. (2016). Tecnología para la evaluacióndel desempeño individual alineada al desempeño organizacional: aplicación en organizaciones turísticas de Holguín. La HaBana: Editorial Universitaria. Obtenido de https://ebookcentral.proquest.com/lib/utmachalasp/reader.action?docI $\mathrm{D}=4946042$ \&query=evaluacion $\% 252 \mathrm{Bde} \% 252 \mathrm{Bdesempe} \% 2525 \mathrm{C} 3$ \%2525B1oMalhotra, N. (2008). Investigación de mercados. México: Pearson Educación.

11. Martinez, F., Sánchez, V., Somohano, F., García , D., \& Marín, S. (2008). Análisis estrategico para el desarrollo de la micro, pequeña ymediana empresa. Pachica, Hidalgo: Universidad Autonoma del Estado de Hidalgo.

12. Matabanchoy, S., Álvarez, K., y Riobamba, O. (2018). Efectos de la evaluación de desempeño en la calidad de vida del trabajador: Revisión del tema entre 2008-2018. Universidad y salud, 176-187. Obtenido de http://www.scielo.org.co/scielo.php?pid=S0124-

$71072019000200176 \&$ scr ipt $=$ sci_abstract\&tlng=es

13. Molina, R. (2011). Propuesta Metodologica de Benchmarking en pymes: PDG Manufacturier. Academia de Ciencias Administrativas, 222-239. Obtenido de

http://www.aeca1.org/pub/on_line/comunicaciones_aal2011/cd/77c.p df

14. Quinn, R. y Rohrbaugh, J. (1983). "A spatial model of effectiveness criteria: towards a competing values approach to organizational analysis". Management Science. 29(3)

15. Villar, M. (2018). Movilizando a las personas y sus recursos humanos para el logro de resultados empresariales. Santiago: RIL Editores. Obtenido de https://ebookcentral.proquest.com/lib/utmachalasp/reader.action?docI $\mathrm{D}=5759143 \&$ query=desempe $\% 25 \mathrm{C} 3 \% 25 \mathrm{~B} 1 \mathrm{o} \% 2$ Blaboral 